\title{
Introduction to molecular biology of influenza A viruses
}

\author{
Bogusław Szewczyk ${ }^{\boxplus}$, Krystyna Bieńkowska-Szewczyk² and Ewelina Król ${ }^{1}$ \\ 1Department of Recombinant Vaccines, Intercollegiate Faculty of Biotechnology, University of Gdansk and Medical University of Gdansk, \\ Gdańsk, Poland; 2Department of Virus Molecular Biology, Intercollegiate Faculty of Biotechnology, University of Gdansk and Medical University \\ of Gdansk, Gdańsk, Poland
}

This minireview presents an overview of current knowledge on virion structure, genome organization and basic events in the development of influenza A virus. The processes of entry, transcription/replication and viral release are described. In this context, the roles of viral proteins (including recently discovered minor polypeptides) in the subsequent stages of viral development are also discussed.

Key words: Orthomyxoviridae, influenza A virus, virion structure, genome, entry, release, new polypeptides

Received: 13 July, 2014; revised: 17 August, 2014; accepted: 18 August, 2014; available on-line: 03 September, 2014

\section{INTRODUCTION}

A number of excellent reviews on influenza viruses has been published in recent years (Taubenberger \& Morens, 2008; Krauss \& Webster, 2010; Medina \& GarciaSastre, 2011; Wahlgren J 2011; Iwasaki \& Pillai, 2014; Spackman, 2014). This article summarizes basic facts on the molecular biology of influenza viruses and it should serve only as a reference article for the remaining articles in this special issue of Acta Biochimica Polonica. For more thorough review of influenza molecular biology we also suggest chapters on Orthomyxoviridae in the latest edition of Fields Virology (Shaw \& Palese, 2013).

Influenza viruses are the enveloped RNA viruses belonging to the relatively small Orthomyxoviridae family. The single-stranded genomic RNA of this family has been termed negative in contrast to mRNA which, by convention, is called positive-strand RNA. At present, Orthomyxoviridae family consists of five genera: Influenzavirus $A$, Influenzavirus $B$, Influenzavirus $C$, Thogovirus and Isavirus. The first four genera were established long time ago. The fifth genus is a relatively new addition to Orthomyxoviridae; it contains an infectious salmon anemia virus, which is a devastating pathogen in fish farms all over the world. It is likely that the sixth genus, Influenzavirus $D$ will be soon created because the virus $\mathrm{C} / \mathrm{OK}$ isolated from swine and cattle, provisionally included in the Influenzavirus $C$ genus, exhibits only low sequence homology to the influenza $C$ viruses (Hause et al., 2014). From the epidemiological point of view it is Influenzavirus $A$ genus that is the most important for humans. The only representative of this genus - influenza A virus is divided into many subtypes. According to the current nomenclature, description of the subtypes includes the host from which it was originally isolated, geographic location of the first isolation, strain number, year of isolation and, in paren- theses, the serological characteristics of two viral proteins: hemagglutinin (HA) and neuraminidase (NA). For the strains isolated from humans, the name of the host is omitted. As an example, A/Sw/Taiwan/1/70 (H1N2) should be read: influenza A strain number 1 , isolated from swine in Taiwan in 1970, which contains hemagglutinin HA1 and neuraminidase NA2. As for today, 18 serotypes of hemagglutinin and 11 serotypes of neuraminidase are described (Tong et al., 2013).

\section{INFLUENZA A VIRION STRUCTURE}

Orthomyxovirus virions are usually close to being spherical, with diameter ranging from $100-200 \mathrm{~nm}$ (Fig. 1). However, some laboratory strains may form extended threadlike structures, which sometimes exceed $1000 \mathrm{~nm}$. The virions are relatively unstable in the environment and influenza viruses are inactivated by heat, dryness, extremes of $\mathrm{pH}$ and detergents (Shaw \& Palese, 2013). The outer lipid layer of influenza viruses originates from the plasma membranes of the host in which the virus was propagated (Nayak et al., 2009). Outside of the lipid envelope, there are about 500 projections/virions in the form of spikes. About $80 \%$ of these projections resemble rods which are composed of hemagglutinin. The remaining projections are in the shape of mushrooms and they are built from molecules of neuraminidase. The viral outer membrane also contains some copies of the small M2 protein that form ion channels in the virion particles. The matrix protein M1, which is the most abundant protein in virions, underlies the lipid layer and plays an important role in the attachment of the ribonucleoprotein (RNP). The RNP core is a complex structure

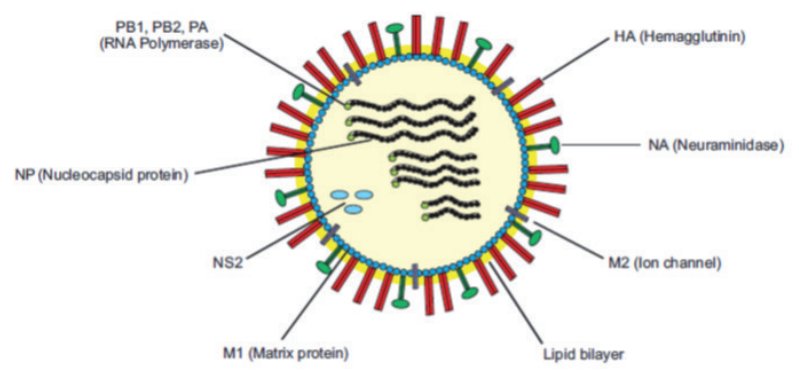

Figure 1. Schematic view of the influenza A virion structure.

e-mail: szewczyk@biotech.ug.gda.pl

Abbreviations: CRNA, complementary copy of the VRNA; HA, hemagglutinin; M1, matrix protein; NA, neuraminidase; NEP, nuclear export protein; RNP, ribonucleoprotein; ssRNA, single-stranded RNA; vRNA, viral RNA. 
composed mostly of the NP protein, which wraps eight different RNA segments of the influenza A genome. Additionally, RNPs contain about 50 copies per virion of RNA-dependent RNA polymerase, which in case of influenza A virus is a complex of three proteins: PB1, PB2 and PA (Noda et al., 2006; Boivin et al., 2010).

\section{INFLUENZA A GENOME ORGANIZATION}

Influenza A's eight segments of single-stranded RNA (ssRNA) of negative polarity contain about $14000 \mathrm{nu}-$ cleotides. Historically, the gene assignment of gene products to particular segments was as follows:

Segment $1-$ PB2

Segment $2-$ PB1

Segment $3-\mathrm{PA}$

Segment 4 - HA

Segment 5 - NP

Segment 6 - NA

Segment $7-$ M1 and M2

Segment 8 - NS1 and NS2/NEP

Recently some new gene products were added to this list. They are described in the last subchapter of this article.

The first three largest segments encode three subunits of the RNA polymerase which, as mentioned above, are found in virions associated with viral RNA. Proteins encoded by segments 4-7 and one protein encoded by segment 8 - NS2/NEP are also found in the viral particle. Only segment 8 encodes a protein, NS1, which is a non-structural protein. The organization of the eight RNA segments inside the virion is not fully understood. Electron microscopy studies indicate that each RNA segment is individually wrapped by the NP protein forming helical structures. The mechanism of packaging of eight different segments into a single virion shell still remains to be elucidated.

\section{PROPAGATION OF INFLUENZA A VIRUS}

Historically, influenza viruses were first isolated from swine in 1930 and from humans three years later. For a few decades embryonated chicken eggs were the only reactors where influenza viruses could be grown. This is still a method of choice for the cultivation of the virus for vaccine production, where massive quantities of the virus are needed. Some subtypes of influenza A do not replicate well in chicken embryos and they require adaptation to growth in the allantoic cavity. The quality of the virus replication in embryonated eggs can be assessed by the ability of the infected allantoic fluid to agglutinate erythrocytes. At present, influenza viruses are often grown in tissue cultures originating from kidney cells (most often these are Madin-Darby canine kidney cells - MDCK) (Govorkova et al., 1999; Tree et al., 2001). However, for most HA subtypes preliminary treatment with trypsin is necessary to produce infectious viral particles. This protease cleaves hemagglutinin into active subunits, which is a prerequisite for the efficient propagation of the virus.

\section{THE ROLE OF PROTEINS ENCODED BY INFLUENZA A GENOME IN THE SUBSEQUENT STAGES OF VIRAL MULTIPLICATION}

Stages of influenza A replication are essentially the same as for the majority of other ssRNA viruses of neg- ative polarity. One can distinguish the following major processes leading to the production of viral progeny:

- Virus adsorption, entry and uncoating

- Messenger RNA synthesis and replication of viral RNA

- Post-transcriptional processing of viral mRNA

- Translation and post-translational processing of viral proteins

- Virus assembly and release from the cells

Each of these processes has one or more viral proteins playing a key role in the accomplishment of the successful outcome of the subsequent steps of viral amplification. However, it has to be pointed out that some cellular factors play a crucial role in allowing the virus to achieve this final aim.

\section{Virus adsorption, entry and uncoating}

Influenza A virus utilizes sialic acids on the surface of cells as viral receptors (Skehel \& Wiley, 2000). Hemagglutinins of influenza viruses replicating in different species show some preference for particular glycosidic linkages of sialic acids. Human viruses' hemagglutinins bind very well to sialic acids linked to galactose by alfa 2,6 glycosidic bond. On the other hand, avian viruses preferentially bind to sialic acids connected with galactose by alfa 2,3 glycosidic bond (Matrosovich et al., 1997; Wilks et al., 2012). Alfa 2,6 linkage of sialic acids to galactose is found mostly in human trachea, while sialic acid alfa 2,3 galactose sugar moieties are found mostly in gut epithelium of birds. This fact explains the preference of different viral subtypes for particular host species, but it should be pointed out that it is a preference for receptor and not absolute specificity. It may be bypassed by high viral inoculum (as was the case with most infections of humans with influenza $\mathrm{H} 5 \mathrm{~N} 1$ ) or by a few point mutations in the hemagglutinin gene.

After binding to the receptor, influenza viruses are internalized in endocytic compartments. The main mechanism is clathrin-mediated endocytosis, although non-clathrin, non-caveolae pathway has been also attributed to influenza entry (Lakadamyali et al., 2004; Grove \& Marsh, 2011). Low pH treatment in endosomes dramatically changes hemagglutinin structure, which becomes susceptible to protease digestion. As a consequence, hemagglutinin is cleaved into HA1 and HA2 subunits, which are connected together by disulfide bonds. The cleaved hemagglutinin undergoes further structural changes, which are evidenced by exposure of the fusion peptide at the $\mathrm{N}$-terminus of the HA2. This in turn leads to the interaction of hemagglutinin with membranes of endosomes. During entry, virions must induce signalling to facilitate successful trafficking via the endocytic pathway (Elbahesh et al., 2014).

As a consequence of these subsequent processes, contents of the virion, that is its genetic material in the form of a ribonucleoprotein complex, are released into cytoplasm of the cell (Stegmann, 2000; Sieczkarski \& Whittaker, 2005). Apart from hemagglutinin, the M2 protein which forms ion channels, also plays an important role in the release of the genetic material from the virion. It mediates the influx of $\mathrm{H}^{+}$ions from the endosome into viral particle. As a result, the interaction between ribonucleoprotein and matrix M1 protein is greatly weakened (Martin \& Helenius, 1991; Pinto \& Lamb, 2006; Wang et al., 2011). Recent findings suggest that all eight segments are transported as one moiety to the nucleus (Chou et al., 2013). 


\section{mRNA synthesis and replication of virion RNA}

RNA synthesis of influenza virus occurs in the nucleus. The genomic segments of the virus never exist as naked RNA and it is the NP protein which wraps viral RNA. Ribonucleoprotein complexes are not able to diffuse passively through nuclear pores, so they must rely on cellular mechanisms of nuclear transport. Proteins belonging to the family of alfa importins recognize nuclear localization signals (NLS) on the NP protein and play an important role in the transport of RNA complexes (Cros \& Palese, 2003).

Once in the nucleus, genomic ssRNA of negative polarity (vRNA) is used as a template for the syntheses of two classes of ssRNAs of positive polarity: messenger RNAs (mRNAs) and full-length complementary copies (cRNAs). Messenger RNAs are the incomplete copies of the template and they are capped and polyadenylated. During transcription, multimeric viral polymerase interacts with the host polymerase II (Engelhardt et al., 2005). This interaction leads to the phenomenon called "cap-snatching", that is the removal of a cap from newly synthetized cellular mRNAs (Plotch et al., 1979; Bouloy et al., 1979; Bouloy et al., 1980; Krug, 1981). The PB2 subunit of viral polymerase is responsible for cap binding. The PB1 subunit for a long time was regarded as an endonuclease cleaving the 5' capped oligonucleotide (10-13 nucleotides long), which serves as a primer for the initiation of transcription. However, recent crystallographic data suggest that endonuclease activity should be rather attributed to the PA subunit (Boivin et al., 2010). This is one of the facts that contradicts the theory that particular activities of the polymerase can be dissected and attributed to a single subunit. It is rather more likely that three subunits of the polymerase act cooperatively and the synthesis of RNAs is a concerted action of all subunits. Transcription continues until approximately 15 nucleotides from the 5' end of the vRNA (Li et al., 2001). Here, a stretch of uridine residues is localized which serves as a signal for polyadenylation (also catalyzed by viral polymerase). Some mRNA transcripts undergo splicing; this phenomenon leads to the increase of the coding capacity of the segments. Splicing was most extensively studied for transcripts originating from segments 7 and 8 . Splicing can be observed in the absence of viral proteins, which suggests that this process is executed by cellular machinery (Lamb \& Lai, 1984).

The switch from mRNA synthesis to the synthesis of full-length complementary copy (cRNA) of the vRNA has been intensively studied by many laboratories. For the initiation of the full-length cRNA, no capped primer is necessary and the cRNA chain is not prematurely terminated and polyadenylated, as is the case of viral mRNA synthesis. The precise mechanism of the switch from one to another mode of RNA synthesis is not fully understood; some theories, which were proposed to explain the switch have been described (Gonzalez \& Ortin, 1999). Obviously, the polymerase subunits may have different contribution to these two processes, but it has been suggested that the soluble NP protein and some host proteins play an important role in this process (Momose et al., 2002).

The next step of replication of influenza genomic segments is the copying of vRNA on the template of positive strand cRNAs. This process also generates fulllength products which assemble with NP and polymerase subunits and form RNP complexes. Such complexes are finally exported into the cytoplasm; the M1 protein and NS2 protein (also called nuclear export protein -
NEP) play a vital role in the translocation of these macromolecular structures (O’Neill et al., 1998; Huang et al., 2001).

\section{Virus assembly and release}

It has been postulated that assembly of viral components occurs preferentially in lipid rafts (Zhang et al., 2000; Takeda et al., 2003; Leser \& Lamb, 2005). Lipid rafts are non-ionic lipid structures, rich in sphingolipids, which reside inside plasma membranes. This site of assembly of influenza virus is supported by the results of examination of the lipid content of purified viral particles. Additionally, hemagglutinin and neuraminidase glycoproteins are incorporated into the lipid rafts (Kundu et al., 1996; Scheiffele et al., 1997; Rossman \& Lamb, 2011). Before being directed into lipid rafts, these two glycoproteins are posttranslationally modified. The modifications take place in the endoplasmic reticulum and in the Golgi apparatus. In the endoplasmic reticulum these proteins become correctly folded and glycosylated. They are also assembled into oligomers: hemagglutinin into trimers and neuraminidase into tetramers. Subsequent modifications of the glycan chains of these proteins and their esterification with fatty acids occurs in the Golgi apparatus (Veit et al., 1991). Signals for the association of these glycoproteins with lipid rafts are located in their transmembrane domains (Barman et al., 2001). RNPs are probably transported to the region of assembly at the apical plasma membrane independently of other structural proteins. Chou and colleagues concluded on the basis of FISH analysis that eight viral segments gather together in the cytoplasm, which is facilitated by endosomal protein Rab 11. All eight segments probably form a supercomplex before arrival at the plasma membrane binding sites (Chou et al., 2013).

Packaging of eight different RNA segments in the form of RNPs into virion shells is a phenomenon poorly understood, though many models have been proposed (Smith \& Hay, 1982; Enami et al., 1991; Bancroft \& Parslow, 2002). Some facts suggest the presence of packaging signals at both ends of genomic segments, however, the precise sequences or structures responsible for specific packaging are still not well defined.

Both glycoproteins, neuraminidase and hemagglutinin, have affinity for sialic acids but they have opposite effect on the release of the virus from cells. Hemagglutinin anchors the virus to the cell membrane due to its interaction with sialic acid-containing receptors. Neuraminidase is required to remove sialic acids in order to allow virus to leave its host cells. Therefore, there must be a rigid balance between the activities of these two glycoproteins, so that maximum progeny of the virus can be produced.

\section{Evolution of influenza A virus}

The genetic changes of influenza A virus may be introduced by at least three evolutionary mechanisms:

- Changes due to the RNA polymerase errors

- Reassortment

- Recombination

The RNA polymerase of influenza viruses, similarly to other viral RNA polymerases, has no proofreading activity, so the mutation rate for this and other RNA viruses, due to the error-prone reading by RNA polymerases, is much higher than for DNA viruses where polymerases have proofreading properties. Hence, the mutation rate for RNA genomes can be up to $10^{5}$ higher than for a viral DNA genome. Each round of replication may introduce more than one point mutation per replicated genome; these mutations may have neutral, negative or positive effect on virus vi- 
ability. The most apparent effect of these gradual changes resulting from the positive selection is so-called antigenic drift in hemagglutinin and neuraminidase glycoproteins, which are localized on the outside of the virion. The antigenic drift, that is the gradual changes in the antigenicity of these two proteins, may finally lead to the establishment of viral variants which are no longer recognized and neutralized by antibodies produced against the parental strain. Therefore, such new antigenic variants may cause epidemics. These variants are usually dominant strains in human or animal populations for a few years and then they are replaced by new antigenic variants. Though this phenomenon is most evident for hemagglutinin and neuraminidase, obviously other genes undergo the same gradual changes as well, caused by polymerase error-prone copying.

Much more abrupt changes in the genome content are caused by gene reassortment. This phenomenon can be defined as the rearrangement of viral gene segments occurring when a cell is infected with two different genomic variants of influenza A viruses. Reassortment usually takes place in animal species, which can be infected with strains of different origin, e.g. with avian and human strains. Very often it is swine that is such a reactor; after exchange of genomic segments, new strains with drastically changed properties may be established in the population. When hemagglutinin or neuraminidase gene segments undergo reassortment, the phenomenon is termed antigenic shift. It implies that major antigenic changes of these two most antigenic proteins may result in abrupt appearance of new strains, which are not recognized by human or animal immunological systems and they may give rise to pandemics. More detailed description of this unique phenomenon will be given in this issue of Acta Biochimica Polonica in an article describing swine influenza.

RNA recombination (both homologous and non-homologous) is not a common event in the evolution of influenza A viruses, though it probably takes place more often than it was believed in the past. Non-homologous recombination, which is more common, may occur between genes on different segments (Orlich et al., 1994; (Pasick et al., 2005). As the result of recombination, lower pathogenic strain is sometimes converted to a highly pathogenic strain (Hirst et al., 2004; Suarez et al., 2004).

\section{NEW ADDITIONS TO THE LIST OF GENE PRODUCTS CODED FOR BY INFLUENZA A GENOME}

Since the beginning of this century, several new influenza A proteins have been identified. The PB1-F2 protein discovered in 2001 is encoded by an open reading frame near the 5' end of the PB1 gene (Chen et al., 2001). This is a small, about 90 aa long, polypeptide which is found only in some strains of influenza A. PB1-F2 is translated from the fourth AUG codon in the second open reading frame $(+1)$ of the PB1 gene. Apparently, this protein is an inducer of proapoptotic activity and it contributes to viral pathogenesis in mice (Zamarin et al., 2006). PB1-F2 has no polymerase activity, similarly to the third polypeptide encoded by PB1 segment, named PB1-N40. PB1-N40 is an N-terminal 39 aa polypeptide which is translated from the fifth AUG codon in frame with the PB1 start codon (Wise et al., 2009). The virus that lacks PB1-N40 has a slower replication rate, however, the more detailed role of this protein remains to be elucidated.

Novel polypeptides encoded by genomic segment 3 also have been recently identified. The first of them, PA-X, is encoded by an alternative reading frame of PA (Jagger et al., 2012). PA-X modulates host response and viral virulence
(Schrauwen et al., 2014). It is expressed in majority of viral strains, though at a low level. It has been found that loss of PA-X changes the pathogenicity of the 1918 pandemic strain in experimental animals. Recently, two novel polypeptides which are truncated forms of the PA polymerase subunit, have been described (Muramoto et al., 2013). They are translated from the $11^{\text {th }}$ and $13^{\text {th }}$ in frame AUG codons in the PA mRNA. These two polypeptides, PA-N155 and PA-N182, do not exhibit polymerase activity, however mutants lacking these polypeptides replicate less efficiently in cell culture, and they are less pathogenic in mice than wild type viruses.

Still another protein recently discovered is M42, which is an M2-like protein, but with a different extra-cellular domain than M2 (Wise et al., 2012). The list of novel polypeptides encoded by influenza $A$ is not closed. eNP and NS3 are other polypeptides postulated to be coded for by this virus. It remains to be elucidated whether these new additions to the list of influenza proteins play a significant role in viral pathogenicity and fitness.

\section{CONCLUSIONS}

Influenza remains one of the main public health threats of this century. Quickly increasing knowledge on molecular biology of influenza is a great help in search for means of prevention of future epidemics caused by this elusive pathogen. New antiviral drugs and novel approaches to construction of vaccines would not be possible without extensive molecular and bioinformatic data collected during recent years in many laboratories all over the world. We anticipate in the near future further breakthroughs in the prevention and therapy, like universal vaccines and inhibitors of viral transcription/ replication, which may reduce the health burden caused by influenza.

\section{Acknowledgements of financial support}

This work was supported by the Innovative Economy Program, Grant No. WND-POIG.01.01.02-00-007/08 in frame of Vaccine Cluster Consortium (VCC): Institute of Biochemistry and Biophysics, Polish Academy of Sciences, Warsaw, Poland (IBB); Institute of Biotechnology and Antibiotics, Warsaw, Poland (IBA); Department of Recombinant Vaccines, Intercollegiate Faculty of Biotechnology, University of Gdansk and Medical University of Gdansk, Gdansk, Poland (UG); Kucharczyk TE sp. z o.o., Warsaw, Poland (KTE); Institute of Animal Reproduction and Food Research, Polish Academy of Sciences, Olsztyn, Poland (IAR); Department of Poultry Diseases, National Veterinary Research Institute, Pulawy, Poland (PIWet).

\section{LITERATURE}

Bancroft CT, Parslow TG (2002) Evidence for segment-nonspecific packaging of the influenza a virus genome. J Virol 76: 7133-7139.

Barman S, Ali A, Hui EK, Adhikary L, Nayak DP (2001) Transport of viral proteins to the apical membranes and interaction of matrix protein with glycoproteins in the assembly of influenza viruses. $V i$ rus Res 77: 61-69.

Boivin S, Cusack S, Ruigrok RW, Hart DJ (2010) Influenza A virus polymerase: structural insights into replication and host adaptation mechanisms. J Biol Chem 285: 28411-28417.

Bouloy M, Morgan MA, Shatkin AJ, Krug RM (1979) Cap and internal nucleotides of reovirus mRNA primers are incorporated into influenza viral complementary RNA during transcription in vitro. $J$ Virol 32: 895-904.

Bouloy M, Plotch SJ, Krug RM (1980) Both the 7-methyl and the 2'-O-methyl groups in the cap of mRNA strongly influence its abil- 
ity to act as primer for influenza virus RNA transcription. Proc Natl Acad Sci U S A 77: 3952-3956.

Chen W, Calvo PA, Malide D, Gibbs J, Schubert U, Bacik I, Basta S, O’Neill R, Schickli J, Palese P, Henklein P, Bennink JR, Yewdell JW (2001) A novel influenza A virus mitochondrial protein that induces cell death. Nat Med 7: 1306-1312.

Chou YY, Heaton NS, Gao Q, Palese P, Singer RH, Lionnet T (2013) Colocalization of different influenza viral RNA segments in the cytoplasm before viral budding as shown by single-molecule sensitivity FISH analysis. PLoS Pathog 9: e1003358.

Cros JF, Palese P (2003) Trafficking of viral genomic RNA into and out of the nucleus: influenza, Thogoto and Borna disease viruses. Virus Res 95: 3-12.

Elbahesh H, Cline T, Baranovich T, Govorkova EA, Schultz-Cherry S, Russell CJ (2014) Novel roles of focal adhesion kinase in cytoplasmic entry and replication of influenza A viruses. J Virol 88: 6714-6728.

Enami M, Sharma G, Benham C, Palese P (1991) An influenza virus containing nine different RNA segments. Virology 185: 291-298.

Engelhardt OG, Smith M, Fodor E (2005) Association of the influenza A virus RNA-dependent RNA polymerase with cellular RNA polymerase II. J Virol 79: 5812-5818.

Gonzalez S, Ortin J (1999) Distinct regions of influenza virus PB1 polymerase subunit recognize vRNA and cRNA templates. EMBO J 18: 3767-3775.

Govorkova EA, Kodihalli S, Alymova IV, Fanget B, Webster RG (1999) Growth and immunogenicity of influenza viruses cultivated in Vero or MDCK cells and in embryonated chicken eggs. Dev Biol Stand 98: 39-51.

Grove J, Marsh M (2011) The cell biology of receptor-mediated virus entry. J Cell Biol 195:1071-1082.

Hause BM, Collin EA, Liu R, Huang B, Sheng Z, Lu W, Wang D, Nelson EA, Li F. (2014) Characterization of a novel influenza virus strain in cattle and swine: proposal for a new genus in the Orthomyxoviridae family. mBio 5: e00031-14.

Hirst M, Astell CR, Griffith M, Coughlin SM, Moksa M, Zeng T, Smailus DE, Holt RA, Jones S, Marra MA, Petric M, Krajden M, Lawrence D, Mak A, Chow R, Skowronski DM, Tweed SA, Goh S, Brunham RC, Robinson J, Bowes V, Sojonky K, Byrne SK, Li Y, Kobasa D, Booth T, Paetzel M (2004) Novel avian influenza H7N3 strain outbreak, British Columbia. Emerg Infect Dis 10: 2192-2195.

Huang X, Liu T, Muller J, Levandowski RA, Ye Z (2001) Effect of influenza virus matrix protein and viral RNA on ribonucleoprotein formation and nuclear export. Virology 87: 405-416.

Iwasaki A, Pillai PS (2014) Innate immunity to influenza virus infection. Nat Rev Immunol 14: 315-328.

Jagger BW, Wise HM, Kash JC, Walters KA, Wills NM, Xiao YL, Dunfee RL, Schwartzman LM, Ozinsky A, Bell GL, Dalton RM, Lo A, Efstathiou S, Atkins JF, Firth AE, Taubenberger JK, Digard P (2012) An overlapping protein-coding region in influenza A virus segment 3 modulates the host response. Science 337: 199-204.

Krauss S, Webster RG (2010) Avian influenza virus surveillance and wild birds: past and present. Avian Dis 54: 394-398.

Krug RM (1981) Priming of influenza viral RNA transcription by capped heterologous RNAs. Curr Topics Microbiol Immunol 93: 125149.

Kundu A, Avalos RT, Sanderson CM, Nayak DP (1996) Transmembrane domain of influenza virus neuraminidase, a type II protein, possesses an apical sorting signal in polarized MDCK cells. J Virol 70: 6508-6515.

Lakadamyali M, Rust MJ, Zhuang X (2004) Endocytosis of influenza viruses. Microbes Infect 6: 929-936.

Lamb RA, Lai CJ (1984) Expression of unspliced NS1 mRNA, spliced NS2 mRNA, and a spliced chimera mRNA from cloned influenza virus NS DNA in an SV40 vector. Virology 135: 139-147.

Leser GP, Lamb RA (2005) Influenza virus assembly and budding in raft-derived microdomains: a quantitative analysis of the surface distribution of HA, NA and M2 proteins. Virology 342: 215-227.

Li ML, Rao P, Krug RM (2001) The active sites of the influenza cap-dependent endonuclease are on different polymerase subunits. EMBO J 20: 2078-2086.

Martin K, Helenius A (1991) Transport of incoming influenza virus nucleocapsids into the nucleus. I V irol 65: 232-244.

Matrosovich MN, Gambaryan AS, Teneberg S, Piskarev VE, Yamnikova SS, Lvov DK, Robertson JS, Karlsson KA (1997) Avian influenza A viruses differ from human viruses by recognition of sialyloligosaccharides and gangliosides and by a higher conservation of the HA receptor-binding site. Virology 233: 224-234.

Medina RA, Garcia-Sastre A (2011) Influenza A viruses: new research developments. Nat Rev Microbiol 9: 590-603.

Momose F, Naito T, Yano K, Sugimoto S, Morikawa Y, Nagata K (2002) Identification of $\mathrm{Hsp} 90$ as a stimulatory host factor involved in influenza virus RNA synthesis. I Biol Chem 277: 45306-45314.

Muramoto Y, Noda T, Kawakami E, Akkina R, Kawaoka Y (2013) Identification of novel influenza A virus proteins translated from PA mRNA. J Virol 87: 2455-2462.
Nayak DP, Balogun RA, Yamada H, Zhou ZH, Barman S (2009) Influenza virus morphogenesis and budding. Virus Res 143: 147-161.

Noda T, Sagara H, Yen A, Takada A, Kida H, Cheng RH, Kawaoka Y (2006) Architecture of ribonucleoprotein complexes in influenza A virus particles. Nature 439: 490-492.

O’Neill RE, Talon J, Palese P (1998) The influenza virus NEP (NS2 protein) mediates the nuclear export of viral ribonucleoproteins. EMBO J 17: 288-296.

Orlich M, Gottwald H, Rott R (1994) Nonhomologous recombination between the hemagglutinin gene and the nucleoprotein gene of an influenza virus. Virology 204: 462-465.

Pasick J, Handel K, Robinson J, Copps J, Ridd D, Hills K, Kehler H, Cottam-Birt C, Neufeld J, Berhane Y, Czub S (2005) Intersegmental recombination between the haemagglutinin and matrix genes was responsible for the emergence of a highly pathogenic H7N3 avian influenza virus in British Columbia. J Gen Virol 86: 727-731.

Pinto LH, Lamb RA (2006) The M2 proton channels of influenza A and B viruses. J Biol Chem 281: 8997-9000.

Plotch SJ, Bouloy M, Krug RM (1979) Transfer of 5'-terminal cap of globin $\mathrm{mRNA}$ to influenza viral complementary RNA during transcription in vitro. Proc Natl Acad Sci US A 76: 1618-1622.

Rossman JS, Lamb RA (2011) Influenza virus assembly and budding. Virology 411: 229-236.

Scheiffele P, Roth MG, Simons K (1997) Interaction of influenza virus haemagglutinin with sphingolipid-cholesterol membrane domains via its transmembrane domain. EMBO J 16: 5501-5508.

Schrauwen EJ, de Graaf M, Herfst S, Rimmelzwaan GF, Osterhaus AD, Fouchier RA (2014) Determinants of virulence of influenza A virus. Eur I Clin Microbiol Infect Dis 33: 479-490.

Shaw ML, Palese P (2013) Orthomyxoviridae: The viruses and their replication. In: Fields Virology $\sigma^{\text {th }}$ edition. Knipe DM, Howley PM, eds, pp 1691-1740, Lippincott Williams \& Wilkins, Philadelphia.

Sieczkarski SB, Whittaker GR (2005) Viral entry. Curr Top Microbiol Immunol 285: 1-23.

Skehel JJ, Wiley DC (2000) Receptor binding and membrane fusion in virus entry: the influenza hemagglutinin. Annu Rev Biochem 69: 531-569.

Smith GL, Hay AJ (1982) Replication of the influenza virus genome. Virology 118: $96-108$.

Spackman E (2014) A brief introduction to avian influenza virus. Methods Mol Biol 1161: 61-68.

Stegmann T (2000) Membrane fusion mechanisms: the influenza hemagglutinin paradigm and its implications for intracellular fusion. Traffic 1: 598-604.

Suarez DL, Senne DA, Banks J, Brown IH, Essen SC, Lee CW, Manvell RJ, Mathieu-Benson C, Moreno V, Pedersen JC, Panigrahy B, Rojas H, Spackman E, Alexander DJ (2004) Recombination resulting in virulence shift in avian influenza outbreak, Chile. Emerg Infect Dis 10: 693-699.

Takeda M, Leser GP, Russell CJ, Lamb RA (2003) Influenza virus hemagglutinin concentrates in lipid raft microdomains for efficient viral fusion. Proc Natl Acad Sci U S A 100: 14610-14617.

Taubenberger JK, Morens DM (2008) The pathology of influenza virus infections. Annu Rev Pathol 3: 499-522.

Tong S, Zhu X, Li Y, Shi M, Zhang J, Bourgeois M, et al. (27 authors in total) (2013) New World bats harbor diverse influenza A viruses. PLoS Path 9: e1003657.

Tree JA, Richardson C, Fooks AR, Clegg JC, Looby D (2001) Comparison of large-scale mammalian cell culture systems with egg culture for the production of influenza virus A vaccine strains. Vaccine 19: $3444-3450$

Veit M, Kretzschmar E, Kuroda K, Garten W, Schmidt MF, Klenk HD, Rott R (1991) Site-specific mutagenesis identifies three cysteine residues in the cytoplasmic tail as acylation sites of influenza virus hemagglutinin. J Virol 65: 2491-2500.

Wahlgren J (2011) Influenza A viruses: an ecology review. Infect Ecol Epidemiol 1.

Wang J, Qiu JX, Soto C, DeGrado WF (2011) Structural and dynamic mechanisms for the function and inhibition of the M2 proton channel from influenza A virus. Curr Opin Struct Biol 21: 68-80.

Wilks S, de Graaf M, Smith DJ, Burke DF (2012) A review of influenza haemagglutinin receptor binding as it relates to pandemic properties. Vaccine 30: 4369-4376.

Wise HM, Foeglein A, Sun J, Dalton RM, Patel S, Howard W, Anderson EC, Barclay WS, Digard P (2009) A complicated message: Identification of a novel PB1-related protein translated from influenza A virus segment 2 mRNA. I Virol 83: 8021-8031.

Zamarin D, Ortigoza MB, Palese P (2006) Influenza A virus PB1-F2 protein contributes to viral pathogenesis in mice. J Virol 80: 79767983.

Zhang J, Pekosz A, Lamb RA (2000) Influenza virus assembly and lipid raft microdomains: a role for the cytoplasmic tails of the spike glycoproteins. J Virol 74: 4634-4644. 